Paediatric Psychology

Original Research Study

Central Eur J Paed 2020;16(1):69-82

DOI $10.5457 / \mathrm{p} 2005-114.258$

\title{
Appearance Self-Attitudes and Health and Well-Being of Bulgarian Adolescents
}

\author{
Anna Alexandrova-Karamanova \\ Department of Psychology, Institute for Population and Human Studies - Bulgarian Academy of Sciences
}

Correspondence: annaalexandrova@yahoo.com; Tel.: + 3592979 3043; Fax.: + 35928703217

Received: 18 December 2019; Accepted: 15 February 2020

\begin{abstract}
Objective - The study aims to explore the associations between adolescents' appearance self-attitudes and their self-rated health, psychosomatic complaints and psychological well-being. A number of covariates known to impact health and well-being are also being investigated. Materials and Methods - The study utilizes data from the Bulgarian 2013/2014 Health Behaviour in SchoolAged Children (HBSC) study. The national representative sample included 3204 adolescents aged 13 and 15 . The methods used were the Body image feelings and attitudes subscale of the Body Investment Scale (BIS), the HBSC psychosomatic complaints scale, the KIDSCREEN-10 index as a measure of psychological well-being, and single-item measures for appearance satisfaction and self-rated health. Results: Adolescents' appearance satisfaction and positive feelings and attitudes towards body and appearance were significantly associated with better self-rated health, absence of or little psychosomatic complaints and better psychological well-being. These associations remained significant after the addition of a large number of covariates, including sociodemographic characteristics (gender, age, and family affluence); chronic health conditions; health behaviours (healthy eating, physical activity, smoking, alcohol consumption, and dieting to lose weight); overweight/obesity; and peer victimization. Adolescents' appearance self-attitudes explained the largest proportions of the variances in self-rated health, psychosomatic complaints and psychological well-being, compared to the other groups of independent variables. Conclusion - The results demonstrate the great significance of appearance self-attitudes for physical and mental health and well-being in adolescence. They are important for medical practice, health education and health promotion among adolescent populations, where appearance self-attitudes should be considered as crucial risk factors for health.
\end{abstract}

Key Words: Appearance Self-Attitudes • Self-Rated Health • Psychosomatic Complaints • Psychological Well-Being • Adolescents.

\section{Introduction}

There is a core distinction between "outside" and "inside" views of human physical appearance (1). The "outside view" concerns the way one is perceived and treated by others based on his/her observable physical characteristics. The "inside view" is reflected in the concept of "body image" and pertains to the subjective experiences of what one looks like in respect to a wide range of characteristics: facial features and expression, body sizes and shapes, clothing, hair style, make-up, etc. $(1,2)$. Body image is part of the self-image and is defined as a multidimensional construct that incorporates perceptual and attitudinal dimensions (3). The perceptual component refers to the perception of one's body and physical appearance and is usually measured by investigating the accuracy of body size estimation relative to actual size. The attitudinal component refers to the thoughts (cognitions), feelings (affect), and behaviour of a person to his or her own body and appearance. Attitudinal body image is assessed also as a global subjective satisfaction with one's body and appearance (3). In the present study two attitudinal measures of overall physical appearance will be used: subjective satisfaction with overall appearance and feelings and attitudes towards one's body and appearance, which will be 
referred to as "appearance self-attitudes measures" throughout the paper.

Body and appearance dissatisfaction are highly prevalent in industrialized countries and have been identified in children as young as six years old (34). An important factor is the internalization of the ideals for beauty existing within the Western culture, portrayed in the media and communicated in social interactions, which main features are thin body for women and lean and muscular body for men $(2-3,5)$. Not meeting the hardly achievable standards of the appearance ideal leads to body and appearance dissatisfaction, with weight and shape/muscularity concerns being the most often investigated. Women generally have higher levels of appearance dissatisfaction than men, probably because appearance has traditionally been considered much more important for women than for men in the Western culture (e.g. 2-5). Dissatisfaction with body and appearance has been found to be so common among girls and women that the term "normative discontent" has been adopted (6).

The importance of body image and appearance self-attitudes in adolescence is higher than in other developmental periods due to the increased bodyconsciousness related to the many changes that happen in this period: intense physical development and physical changes associated with puberty, identity formation, the development of sexuality and the increased importance of gender roles, the need for being liked by peers and being valued member in peer groups $(2,7)$. Longitudinal studies had found that appearance dissatisfaction has been steadily rising with age in the years between early adolescence and young adulthood (8) or in the years between early and middle adolescence with a peak in middle adolescence (9), after that remaining largely stable (10).

The psychological effect of such high levels of appearance concerns affects self-perceptions and psychosocial functioning and contributes to the initiation and maintenance of a wide range of health risk behaviours aimed at appearance enhancement, especially in adolescents and young adults. These include dieting and different weight control practices, disor- dered eating, smoking for appetite control, excessive exercise, anabolic steroid use and muscle dysmorphia (a form of body dysmorphic disorder), and unnecessary cosmetic surgery $(2-3,7,11-13)$. Engaging in such behaviours may be detrimental to adolescents' physical and mental health and well-being. For example, extreme or prolonged efforts for weight reduction are related to iron and calcium deficiencies, growth retardation, delay in puberty, menstrual disorders and osteoporosis in girls, low self-esteem and negative self-image, anxiety, irritability, concentration difficulties, sleep problems, depression, suicidal ideation and suicide attempts (12, 14-16).

Another way in which appearance is related to health is through appearance-related victimization (bullying) in school-aged children and adolescents (2). Appearance (including weight, shape, clothing, face, height, wearing glasses) is identified in recent studies as the leading cause for bullying in adolescence (reported by $23 \%-51 \%$ of participants) (1719). Appearance-related bullying is an important risk factor in the etiology of disordered eating (2021 ), body dysmorphic disorder (22) and cosmetic surgery for enhancement of appearance (23), as well as for poor physical (worse self-rated health, psychosomatic complaints, sleep problems) and mental health (internalizing problems like depression, anxiety, low self-esteem, suicidal ideation and suicide attempts; externalizing problems like violence and aggression, alcohol and drug use, truancy; psychotic symptoms later in life) $(17,20,24)$. Except through health behaviours and appearance-related bullying, body and appearance dissatisfaction has also been found to be directly associated with aspects of physical and mental health and well-being in several studies with adolescents globally (e.g. 9, 25-26).

The aim of the present study is to explore the associations between Bulgarian adolescents' appearance self-attitudes and their self-rated health, psychosomatic complaints and psychological wellbeing. Though some evidence that appearance self-perceptions and self-attitudes are significantly related to aspects of physical and mental health and well-being in adolescence has been obtained in recent years, these relationships are still a topic that 
is rarely explored. It is expected that more positive appearance self-evaluations will be associated with better self-rated health, absence of or little psychosomatic complaints, and better psychological well-being. A number of covariates known to impact health and well-being will also be investigated. These include adolescents' socio-demographic characteristics such as gender, age and socioeconomic status; existing chronic health conditions; health behaviours such as healthy eating, physical activity, smoking, alcohol consumption, and dieting to lose weight; overweight/obesity; and peer victimization.

\section{Methods}

\section{The Health Behaviour in School-Aged Children study}

This study utilizes data from the Bulgarian 2013/2014 Health Behaviour in School-Aged Children (HBSC) study. The HBSC study is a WHO collaborative cross-national survey of school-aged children's health and well-being, examined in their social context, conducted every four years in over 40 countries in Europe and North America (27). Data are collected in all participating countries through self-report school-based surveys using a standard methodology described in detail in the HBSC 2013/2014 International Study Protocol (28).

The HBSC questionnaire collects data in multiple areas like health behaviours and health outcomes (eating, physical activity, body image, weight control, overweight and obesity); risk health behaviours (smoking, alcohol and cannabis use, sexual behaviour); bullying and cyberbullying; health and well-being (self-rated health, life satisfaction, psychological well-being, psychosomatic complaints, mental health problems); family relationships and family support; peer relationships and peer support; perceived school performance, school-related stress, and support from teachers and classmates; socio-economic status.

\section{Sampling and Procedure}

Each country uses random sampling to select a proportion of adolescents aged 11, 13 and 15 years, aiming to achieve representative samples of about 1500 adolescents in each age group. In Bulgaria sampling was based on a list of classes, including all 5, 7 and 9 grades - the three grades/school years that most closely match 11-, 13- and 15-year olds. The stratification unit was region (there are 28 regions of the country) and representative sample was achieved through random sampling in each region. The sample included 311 classes from 163 schools. Only one school refused to participate in the study. Data were collected in the schools following permission from the Ministry of Education and Science of Bulgaria. All students who were present and whose parents had given written consent completed the paper-and-pencil questionnaire. The response rate for the three age groups was $77.0 \%$ for 11-year olds, $77.7 \%$ for 13 -year olds, and $80.7 \%$ for 15-year olds. The final sample comprised 4796 students, of whom 2523 (52.6\%) were male and $2273(47.4 \%)$ were female. The 11-year olds were 1592 (33.2\%), the 13-year olds - 1554 (32.4\%), and the 15-year-olds - 1650 (34.4\%). As the appearance self-attitudes measures were used only in the 13- and 15-age groups, solely the data for 13and 15-year old Bulgarian adolescents $(\mathrm{N}=3204)$ were used in the present study.

\section{Materials}

\section{Independent Variables: Appearance Self-Attitudes}

The independent variables included two appearance self-attitudes measures. The first one was a single item measure of appearance (dis)satisfaction, which examined adolescents' self-evaluated overall appearance by a 5-point scale, ranging from (1) not at all good looking to (5) very good looking. The second measure was the 6-item Body image feelings and attitudes subscale of the Body Investment Scale - BIS (29). It examined positive and negative feelings and attitudes towards one's body and appearance through a 5-point Likert scale ranging from (1) I do not agree at all to (5) strongly agree (higher values meaning more positive feelings and attitudes towards one's body and appearance). The scale included the following statements: I am frustrated with my physi- 
cal appearance, I am satisfied with my appearance, I hate my body, I feel comfortable with my body, I feel anger toward my body, and I like my appearance in spite of its imperfections. The Body Investment Scale has demonstrated good psychometric properties (29). Within the Bulgarian 2013/2014 HBSC study Cronbach's Alpha based on standardized items for the Body image feelings and attitudes scale was 0.83 , showing high reliability.

\section{Dependent Variables: Health and Well-Being}

The dependent variables included three measures, assessing adolescents' physical and mental health and well-being: self-rated health, psychosomatic complaints and psychological well-being. Self-rated health was examined through a single item 4-point measure, evaluating adolescents' general health as (1) poor, (2) fair, (3) good or (4) excellent. Psychosomatic complaints were assessed through the Psychosomatic complaints scale - a non-clinical measure of mental and physical health, developed by the HBSC research network, which examines the frequency of eight physical and psychological symptoms: headache, stomachache, backache, feeling low, irritability or bad temper, feeling nervous, difficulties in getting to sleep, and feeling dizzy, on a 5-point scale, ranging from (1) about every day to (5) rarely or never (higher values meaning absence of or little psychosomatic complaints). In the Bulgarian 2013/2014 HBSC study the Psychosomatic complaints scale showed good internal consistency, with Cronbach's Alpha based on standardized items being 0.82.

Psychological well-being was assessed through the KIDSCREEN-10 index (30), a 10-item nonclinical measure of adolescents' psychological wellbeing with 5-point Likert scale response categories. It covers affective (e.g. Have you felt sad?), cognitive (e.g. Have you been able to pay attention?), psycho-vegetative (e.g. Have you felt full of energy?), and psychosocial aspects (e.g. Have you got on well at school?) of mental health (higher values meaning better psychological well-being). The KIDSCREEN-10 index is the shortest tool from the family of instruments developed and normal- ized for surveying health-related quality of life in children and adolescents in 13 European countries within an EU funded project (31). The index has a strong theoretical foundation and is based on the probabilistic Rasch Measurement Model. It provides good discriminatory power along the psychological well-being continuum, has good internal consistency (Cronbach's Alpha based on standardized items in the present study $=0.82$ ) and good test-retest reliability (28).

\section{Covariates}

The present study also controlled for the potential effects of several types of covariates: adolescents' socio-demographic characteristics, existing chronic health conditions, health behaviours and health outcomes, and peer victimization. Socio-demographic characteristics included adolescents' gender (male/female), age (13-year olds and 15-year olds), and socioeconomic status. Socioeconomic status was operationalized through the Family Affluence Scale, third revision (FAS III) $(28,32)$. The Family Affluence Scale is a brief assets-based measure, developed by the HBSC research network as a suitable indicator for assessing family wealth through child or adolescent self-report. Its third revision includes 6 items: number of computers owned by the family, number of cars, number of bathrooms, number of travels/holidays abroad, having an own bedroom, and having a dishwasher. The Family Affluence Scale III had shown high test-retest reliability ( $\mathrm{r}=0.90)$ and consistency between child and parent report $(r=0.80)(28,32)$. In the Bulgarian 2013/2014 HBSC study the scale's internal consistency was little low (Cronbach's Alpha based on standardized items $=0.62$ ), which seemed to be due to the lower correlations between having an own bedroom (which is not very typical for Bulgaria) and the other family wealth items.

The existence of chronic health conditions was explored through a single dichotomous item, assessing if the adolescents had been diagnosed with chronic diseases or disability.

The health behaviours which were considered as covariates in the present study included healthy eat- 
ing, physical activity, smoking, alcohol consumption, and dieting to lose weight. Cannabis use was not included in the analyses, as data about this behaviour are collected only in the 15-year old group.

The Healthy eating scale comprised two items, exploring the frequency of fruits and vegetables consumption per week through a 7-point scale, ranging from (1) never to (7) every day, more than once. Cronbach's Alpha based on standardized items for Healthy eating was 0.68 , indicating good internal consistency for a scale with just two items. A measure of physical activity consistent with the current international guidelines for daily physical activity of adolescents was used. Participants were asked on how many days during the past 7 days their total amount of physical activity was at least 60 minutes per day. Smoking and alcohol consumption were evaluated as the number of days on which adolescents smoked or drank alcohol during the last 30 days. There were 7 response categories, ranging from (1) never to (7) 30 days. Within the present study dichotomous variables were created, representing the proportions of adolescents which have, and which haven't engaged in these behaviours in the last 30 days. Adolescents also reported if they were dieting or doing something else at present in order to lose weight.

The health outcome overweight/obesity was also included as a covariate. Self-reported weight and height data were collected and body mass index (BMI) was calculated. A dichotomous variable, representing the proportions of adolescents being overweight/obese and being underweight/normal weight was created based on the IOTF age- and gender- specific cut-offs (33). Peer victimization was evaluated as the frequency of being bullied at school in the past couple of months. The response categories ranged from (1) I haven't been bullied to (5) several times a week.

\section{Ethics Statement}

The study received approval from the Ministry of Education and Science of Bulgaria. All requirements of the International Study Protocol (28) were met. The HBSC research network accepted Bulgaria's data and included them in the International Report from the 2013/2014 survey (27).

\section{Statistical Analyses}

All statistical analyses were done by means of IBM SPSS Statistics 22. Descriptive statistics were used to present proportions of adolescents for the categorical variables and to define means and standard deviations for the continuous variables. To test the association between appearance self-attitudes and health and well-being, three multiple hierarchical linear regressions were performed with self-rated health, psychosomatic complaints and psychological well-being as the outcome variables. In the first block independent variables were the two appearance self-attitudes measures - appearance satisfaction and feelings and attitudes towards body and appearance, representing the unadjusted association. In the second block of independent variables the socio-demographic variables gender, age and family affluence were added, and in the third block the variable chronic conditions was added. The fourth block of independent variables included the health behaviours healthy eating, physical activity, smoking, alcohol consumption, and dieting to lose weight, as well as being overweight/obese. In the fifth block of independent variables peer victimization was added. All categorical variables were dummy coded. Gender and age were dummy coded with male gender and 13-year olds being the reference categories (coded with zero). For the dichotomous yes/no variables "no" was the reference category.

\section{Results}

As required by the HBSC research protocol, participants were almost equally distributed across gender and age group (Table 1). About 11\% of the adolescents had chronic health conditions. About $18 \%$ were overweight or obese, and $20 \%$ were dieting to lose weight. About $20 \%$ of the adolescents had smoked and about $46 \%$ had consumed alcohol in the last 30 days. 
Table 1. Descriptive Statistics for Categorical Variables:

Demographic Characteristics, Chronic Conditions,

Health Behaviours and Health Outcomes in Bulgarian Adolescents

\begin{tabular}{|c|c|c|}
\hline \multicolumn{2}{|c|}{ Categorical variables } & \multirow{2}{*}{$\frac{N(\%)}{1740(54.3)}$} \\
\hline \multirow{2}{*}{ Gender } & Male & \\
\hline & Female & $1464(45.7)$ \\
\hline \multirow{2}{*}{$\begin{array}{l}\text { Age } \\
\text { (years) }\end{array}$} & 13 & $1554(48.5)$ \\
\hline & 15 & $1650(51.5)$ \\
\hline \multirow{2}{*}{$\begin{array}{l}\text { Chronic } \\
\text { conditions }\end{array}$} & No & $2748(89.4)$ \\
\hline & Yes & $327(10.6)$ \\
\hline \multirow{2}{*}{ Smoking } & No & $2490(80.2)$ \\
\hline & Yes & $614(19.8)$ \\
\hline \multirow{2}{*}{$\begin{array}{l}\text { Alcohol } \\
\text { consumption }\end{array}$} & No & $1629(53.6)$ \\
\hline & Yes & $1413(46.4)$ \\
\hline \multirow{2}{*}{$\begin{array}{l}\text { Dieting to } \\
\text { lose weight }\end{array}$} & No & $2549(79.8)$ \\
\hline & Yes & $644(20.2)$ \\
\hline \multirow{2}{*}{$\begin{array}{l}\text { Overweight/ } \\
\text { obesity }\end{array}$} & No & $2434(82.3)$ \\
\hline & Yes & $522(17.7)$ \\
\hline
\end{tabular}

Most of the participants had medium family affluence $(63.6 \%)$. With low family affluence were $20.3 \%$, and with high family affluence $-16.1 \%$. This was reflected in the mean value, which was about the average (mean of 6.73 within a $0-13$ range) (Table 2). Bulgarian adolescents had rather positive feelings and attitudes towards their body and appearance (mean above the average - 22.39 within a 6-30 range) and were rather satisfied with their appearance (mean above the average - 3.59 within a 1-5 range) (Table 2). Regarding appearance satisfaction, $10.6 \%$ of the adolescents thought they were not very good/not at all good looking, $38.8 \%$ thought that their looks were about average, and $50.6 \%$ thought they were quite good/very good looking.

Bulgarian adolescents' self-rated health was evaluated as very good (mean of 3.43 within a $1-4$ range). Only $0.7 \%$ of the adolescents rated their health as poor and $4.4 \%$ as fair, $46.1 \%$ rated it as good and $48.7 \%$ - as excellent. Results indicated absence of or little psychosomatic complaints (mean of 30.73 within an 8-40 range) and rather good psychological well-being (mean of 37.10 within a 10-50 range) (Table 2). The mean for healthy eating
Table 2. Descriptive Statistics for Continuous Variables:

Family Affluence, Appearance Self-Attitudes, Health and Well-Being, Health Behaviours, and Peer Victimization in Bulgarian Adolescents

\begin{tabular}{lll}
\hline Continuous Variables & Range & Mean \pm SD \\
\hline Family affluence & $0-13$ & $6.73 \pm 2.60$ \\
\hline Appearance satisfaction & $1-5$ & $3.59 \pm 1.01$ \\
\hline Feelings and attitudes towards appearance & $6-30$ & $22.39 \pm 5.16$ \\
\hline Self-rated health & $1-4$ & $3.43 \pm 0.61$ \\
\hline Psychosomatic complaints & $8-40$ & $30.73 \pm 6.91$ \\
\hline Psychological well-being & $10-50$ & $37.10 \pm 7.05$ \\
\hline Healthy eating & $2-14$ & $9.60 \pm 2.78$ \\
\hline Physical activity & $0-7$ & $4.19 \pm 2.20$ \\
\hline Peer victimization & $1-5$ & $1.58 \pm 1.05$ \\
\hline
\end{tabular}

showed that adolescents ate fruits and vegetables often, but not daily. Their total physical activity was 60 minutes or more 4 days a week on average. Most of the adolescents (67.7\%) had never been bullied at school, which was reflected in the low mean value (Table 2). Eight percent were bullied once or several times a week.

The results from the three multiple hierarchical linear regressions with self-rated health, psychosomatic complaints and psychological well-being as the dependent variables are presented in tables 3-5. In the first multiple hierarchical linear regression the dependent variable was self-rated health and five blocks of independent variables were entered. The results showed that in Model 1 the two appearance self-attitudes variables were significantly positively associated with self-rated health, meaning that appearance satisfaction and positive feelings and attitudes towards one's body and appearance were significantly associated with better health. The proportion of the explained variance in self-rated health was 9\% (Table 3). In Model 2, the addition of the socio-demographic characteristics gender, age and family affluence led to a significant increase in the total variance explained by $1 \%$. The associations of the socio-demographic characteristics with self-rated health were significant, with female gender and older age (15-year old group) being associated with worse self-rated health, and higher family affluence being associated with better 
self-rated health. In Model 3 the variable chronic conditions was added, which increased the total explained variance by $2 \%$. Having chronic conditions was negatively associated with self-rated health. In Model 4 the health behaviours and health outcomes variables were entered, which increased the total explained variance in self-rated health by $3 \%$. Significant associations with self-rated health were found for healthy eating, physical activity, smoking and alcohol consumption, with the two healthy behaviours being positively associated with self-rated health and the two unhealthy behaviours - negatively associated with self-rated health. In Model 4 the association between age and self-rated health became insignificant. In Model 5 peer victimiza- tion was added, but its association with self-rated health was insignificant.

When adding all the covariates in Models 2 to 5 , the associations between the two appearance selfattitudes variables and self-rated health remained significant. The standardized regression coefficients beta in Model 5 showed that the appearance selfattitudes variables as well as having chronic conditions had the strongest associations with self-rated health. The two appearance self-attitudes variables explained the largest proportion of the variance in self-rated health compared to the other groups of independent variables (Table 3 ).

The multiple hierarchical linear regression with psychosomatic complaints as the dependent vari-

Table 3. Multiple Hierarchical Linear Regression of the Association between Appearance Self-Attitudes and Self-Rated Health in Bulgarian Adolescents

\begin{tabular}{|c|c|c|c|c|c|c|c|c|c|c|c|c|c|c|c|}
\hline \multirow{2}{*}{ Independent variables } & \multicolumn{3}{|c|}{ Model 1} & \multicolumn{3}{|c|}{ Model 2} & \multicolumn{3}{|c|}{ Model 3} & \multicolumn{3}{|c|}{ Model 4} & \multicolumn{3}{|c|}{ Model 5} \\
\hline & B & SE & $\beta$ & B & SE & $\beta$ & $\mathrm{B}$ & SE & $\beta$ & B & SE & $\beta$ & B & SE & $\beta$ \\
\hline \multicolumn{16}{|l|}{ Appearance self-attitudes } \\
\hline Appearance satisfaction & $0.11^{\ddagger}$ & 0.01 & 0.17 & $0.10^{\ddagger}$ & 0.01 & 0.16 & $0.10^{\ddagger}$ & 0.01 & 0.16 & $0.09^{\ddagger}$ & 0.01 & 0.15 & $0.09^{\ddagger}$ & 0.01 & 0.15 \\
\hline $\begin{array}{l}\text { Feelings and attitudes } \\
\text { towards appearance }\end{array}$ & $0.02^{\ddagger}$ & 0.00 & 0.17 & $0.02^{\ddagger}$ & 0.00 & 0.16 & $0.02^{\ddagger}$ & 0.00 & 0.15 & $0.02^{\ddagger}$ & 0.00 & 0.13 & $0.02^{\ddagger}$ & 0.00 & 0.13 \\
\hline \multicolumn{16}{|c|}{ Socio-demographic characteristics } \\
\hline Gender & - & - & - & $-0.08 \dagger$ & 0.02 & -0.07 & $-0.07^{\dagger}$ & 0.02 & -0.06 & $-0.06^{*}$ & 0.03 & -0.05 & $-0.07^{*}$ & 0.03 & -0.05 \\
\hline Age & - & - & - & $-0.06^{*}$ & 0.02 & -0.05 & -0.06 & 0.02 & -0.05 & 0.00 & 0.03 & 0.00 & -0.00 & 0.03 & -0.00 \\
\hline Family affluence & - & - & - & $0.01 \dagger$ & 0.01 & 0.05 & $0.01^{\dagger}$ & 0.01 & 0.06 & $0.01^{*}$ & 0.01 & 0.05 & $0.01^{*}$ & 0.01 & 0.04 \\
\hline Chronic conditions & - & - & - & - & - & - & $-0.30^{\ddagger}$ & 0.04 & -0.15 & $-0.30^{\ddagger}$ & 0.04 & -0.15 & $-0.30^{\ddagger}$ & 0.04 & -0.15 \\
\hline \multicolumn{16}{|c|}{ Health behaviours \& health outcomes } \\
\hline Healthy eating & - & - & - & - & - & - & - & - & - & $0.02^{\dagger}$ & 0.01 & 0.07 & $0.02^{\dagger}$ & 0.01 & 0.07 \\
\hline Physical activity & - & - & - & - & - & - & - & - & - & $0.03^{\ddagger}$ & 0.01 & 0.10 & $0.03^{\ddagger}$ & 0.01 & 0.10 \\
\hline Smoking & - & - & - & - & - & - & - & - & - & $-0.13^{\ddagger}$ & 0.03 & -0.09 & $-0.13^{\ddagger}$ & 0.03 & -0.08 \\
\hline Alcohol consumption & - & - & - & - & - & - & - & - & - & $-0.07^{\dagger}$ & 0.03 & -0.06 & $-0.07^{\dagger}$ & 0.03 & -0.06 \\
\hline Dieting to lose weight & - & - & - & - & - & - & - & - & - & 0.05 & 0.03 & 0.03 & 0.05 & 0.03 & 0.03 \\
\hline Overweight/obesity & - & - & - & - & - & - & - & - & - & -0.02 & 0.03 & -0.01 & -0.01 & 0.03 & -0.01 \\
\hline Peer victimization & - & - & - & - & - & - & - & - & - & - & - & - & -0.02 & 0.01 & -0.04 \\
\hline \multicolumn{16}{|l|}{ Model summary } \\
\hline F total & \multicolumn{3}{|c|}{$119.80^{\ddagger}$} & \multicolumn{3}{|l|}{$53.22^{\ddagger}$} & \multicolumn{3}{|l|}{$55.95^{\ddagger}$} & \multicolumn{3}{|l|}{$36.02^{\ddagger}$} & \multicolumn{3}{|l|}{$33.58^{\ddagger}$} \\
\hline $\mathrm{R}$ & \multicolumn{3}{|c|}{0.30} & \multicolumn{3}{|l|}{0.32} & \multicolumn{3}{|l|}{0.35} & \multicolumn{3}{|l|}{0.39} & \multicolumn{3}{|l|}{0.40} \\
\hline Adjusted $\mathrm{R}^{2}$ & \multicolumn{3}{|l|}{0.09} & \multicolumn{3}{|l|}{0.10} & \multicolumn{3}{|l|}{0.12} & \multicolumn{3}{|l|}{0.15} & \multicolumn{3}{|l|}{0.15} \\
\hline$\Delta \mathrm{R}^{2}$ & \multicolumn{3}{|l|}{0.09} & \multicolumn{3}{|l|}{0.01} & \multicolumn{3}{|l|}{0.02} & \multicolumn{3}{|l|}{0.03} & \multicolumn{3}{|l|}{0.00} \\
\hline
\end{tabular}

${ }^{\star} \mathrm{P}<0.05 ;{ }^{\dagger} \mathrm{P}<0.01 ;{ }^{\ddagger} \mathrm{P}<0.001 ; \mathrm{B}=$ The unstandardized regression coefficient; $\mathrm{SE}=$ The standard error of the regression coefficient; $\beta=$ The standardized regression coefficient; $\mathrm{F}=$ The test of overall model's significance; $\mathrm{R}=$ The multiple correlation coefficient; Adjusted $\mathrm{R}^{2}=$ The adjusted coefficient of multiple determination; $\Delta \mathrm{R}^{2}=$ The change in $\mathrm{R}^{2}$. 
able showed that in Model 1 the two appearance self-attitudes variables were significantly positively associated with psychosomatic complaints, meaning that appearance satisfaction and positive feelings and attitudes towards one's body and appearance were significantly associated with absence of or little psychosomatic complaints. The proportion of the explained variance in psychosomatic complaints was 9\% (Table 4). In Model 2, the addition of the socio-demographic characteristics gender, age and family affluence led to a significant increase in the total variance explained by $5 \%$. The associations of the socio-demographic characteristics with psychosomatic complaints were significant, with female gender, older age (15-year old group) and higher family affluence being associated with more psychosomatic complaints. In Model 3 the variable chronic conditions was added, which increased the total explained variance in psychosomatic complaints by $2 \%$. Having chronic conditions was associated with more psychosomatic complaints. In Model 4 the health behaviours and health outcomes variables were entered, which increased the total explained variance by $4 \%$. Significant associations with psychosomatic complaints were found for smoking, alcohol consumption, dieting to lose weight and overweight/obesity, with engaging in the three behaviours being associated with more psychosomatic complaints and being overweight/ obese - with absence of or little psychosomatic complaints. In Model 4 the association between age and psychosomatic complaints became insignificant. In Model 5 peer victimization was added, which increased the proportion of total explained variance by $2 \%$. Being bullied at school was associated with more psychosomatic complaints.

When adding all the covariates in Models 2 to 5 , the associations between the two appearance selfattitudes variables and psychosomatic complaints remained significant. The standardized regression coefficients beta in Model 5 showed that feelings and attitudes towards one's body and appearance had the second strongest association with psychosomatic complaints after gender (psychosomatic complaints had strong associations also with peer victimization, chronic conditions, smoking and alcohol consumption). The two appearance selfattitudes variables explained the largest proportion of the variance in psychosomatic complaints compared to the other groups of independent variables (Table 4).

The multiple hierarchical linear regression with psychological well-being as the dependent variable indicated that in Model 1 the two appearance selfattitudes variables were significantly positively associated with psychological well-being, meaning that appearance satisfaction and positive feelings and attitudes towards one's body and appearance were significantly associated with better psychological well-being. The proportion of the explained variance in psychological well-being was $18 \%$ (Table 5). In Model 2, the addition of the socio-demographic characteristics gender, age and family affluence led to a significant increase in the total variance explained by $4 \%$. The associations of the socio-demographic characteristics with psychological well-being were significant, with female gender and older age (15-year old group) being associated with worse psychological well-being and higher family affluence being associated with better psychological well-being. In Model 3 the variable chronic conditions was added, but this led to increase in the total explained variance by less than one percent. Having chronic conditions was associated with worse psychological well-being. In Model 4 the health behaviours and health outcomes variables were entered, which increased the total explained variance in psychological well-being by $5 \%$. Significant associations with psychological well-being were found for healthy eating, physical activity, smoking, alcohol consumption, and overweight/obesity, with the two healthy behaviours and overweight/ obesity being associated with better psychological well-being and the two unhealthy behaviours being associated with worse psychological well-being. In Model 5 peer victimization was added, which increased the proportion of total explained variance by $2 \%$. Being bullied at school was associated with worse psychological well-being. 


\begin{tabular}{|c|c|c|c|c|c|c|c|c|c|c|c|c|c|c|c|}
\hline \multirow{2}{*}{ Independent variables } & \multicolumn{3}{|c|}{ Model 1} & \multicolumn{3}{|c|}{ Model 2} & \multicolumn{3}{|c|}{ Model 3} & \multicolumn{3}{|c|}{ Model 4} & \multicolumn{3}{|c|}{ Model 5} \\
\hline & B & SE & $\beta$ & B & SE & $\beta$ & B & SE & $\beta$ & B & SE & $\beta$ & B & SE & $\beta$ \\
\hline \multicolumn{16}{|c|}{ Appearance self-attitudes } \\
\hline Appearance satisfaction & $0.43^{\dagger}$ & 0.16 & 0.06 & $0.37^{*}$ & 0.16 & 0.05 & $0.35^{*}$ & 0.15 & 0.05 & $0.39^{*}$ & 0.15 & 0.06 & $0.35^{*}$ & 0.15 & 0.05 \\
\hline $\begin{array}{l}\text { Feelings and attitudes } \\
\text { towards appearance }\end{array}$ & $0.36^{\ddagger}$ & 0.03 & 0.27 & $0.30^{\ddagger}$ & 0.03 & 0.23 & $0.29^{\ddagger}$ & 0.03 & 0.22 & $0.26^{\ddagger}$ & 0.03 & 0.20 & $0.30 \ddagger$ & 0.03 & 0.17 \\
\hline \multicolumn{16}{|c|}{ Socio-demographic characteristics } \\
\hline Gender & - & - & - & $-2.96^{\ddagger}$ & 0.27 & -0.22 & $-2.85^{\ddagger}$ & 0.27 & -0.21 & $-2.58^{\ddagger}$ & 0.28 & -0.19 & $-2.73^{\ddagger}$ & 0.27 & -0.20 \\
\hline Age & - & - & - & $-0.81^{\dagger}$ & 0.26 & -0.06 & $-0.80^{\dagger}$ & 0.26 & -0.06 & 0.13 & 0.27 & 0.01 & -0.04 & 0.26 & -0.00 \\
\hline Family affluence & - & - & - & $-0.15 \dagger$ & 0.05 & -0.06 & $-0.13^{\dagger}$ & 0.05 & -0.05 & -0.10 & 0.05 & -0.04 & $-0.10^{*}$ & 0.05 & -0.04 \\
\hline Chronic conditions & - & - & - & - & - & - & $-3.15^{\ddagger}$ & 0.42 & -0.14 & $-3.03^{\ddagger}$ & 0.41 & -0.14 & $-2.83^{\ddagger}$ & 0.40 & -0.13 \\
\hline \multicolumn{16}{|c|}{ Health behaviours \& health outcomes } \\
\hline Healthy eating & - & - & - & - & - & - & - & - & - & 0.03 & 0.05 & 0.01 & 0.03 & 0.05 & 0.01 \\
\hline Physical activity & - & - & - & - & - & - & - & - & - & 0.03 & 0.06 & 0.01 & 0.03 & 0.06 & 0.01 \\
\hline Smoking & - & - & - & - & - & - & - & - & - & $-2.22^{\ddagger}$ & 0.34 & -0.13 & $-2.11^{\ddagger}$ & 0.34 & -0.12 \\
\hline Alcohol consumption & - & - & - & - & - & - & - & - & - & $-1.71^{\ddagger}$ & 0.27 & -0.13 & $-1.79^{\ddagger}$ & 0.27 & -0.13 \\
\hline Dieting to lose weight & - & - & - & - & - & - & - & - & - & $-0.81^{*}$ & 0.33 & -0.05 & $-0.88^{\dagger}$ & 0.32 & -0.05 \\
\hline Overweight/obesity & - & - & - & - & - & - & - & - & - & $1.13^{\dagger}$ & 0.35 & 0.06 & $1.17^{\dagger}$ & 0.35 & 0.06 \\
\hline Peer victimization & - & - & - & - & - & - & - & - & - & - & - & - & $-1.01^{\ddagger}$ & 0.13 & -0.15 \\
\hline \multicolumn{16}{|l|}{ Model summary } \\
\hline F total & 123.60 & & & $79.68^{\ddagger}$ & & & $77.40^{\ddagger}$ & & & $51.39^{\ddagger}$ & & & $53.76^{\ddagger}$ & & \\
\hline $\mathrm{R}$ & 0.31 & & & 0.38 & & & 0.41 & & & 0.46 & & & 0.48 & & \\
\hline Adjusted $\mathrm{R}^{2}$ & 0.09 & & & 0.14 & & & 0.16 & & & 0.20 & & & 0.23 & & \\
\hline$\Delta \mathrm{R}^{2}$ & 0.09 & & & 0.05 & & & 0.02 & & & 0.04 & & & 0.02 & & \\
\hline
\end{tabular}

${ }^{*} \mathrm{P}<0.05 ;{ }^{\dagger} \mathrm{P}<0.01 ;{ }^{\ddagger} \mathrm{P}<0.001 ; \mathrm{B}=$ The unstandardized regression coefficient; $\mathrm{SE}=$ The standard error of the regression coefficient; $\beta=$ The standardized regression coefficient; F=The test of overall model's significance; $\mathrm{R}=$ The multiple correlation coefficient; Adjusted $\mathrm{R}^{2}=$ The adjusted coefficient of multiple determination; $\Delta \mathrm{R}^{2}=$ The change in $\mathrm{R}^{2}$.

When adding all the covariates in Models 2 to 5 , the associations between the two appearance selfattitudes variables and psychological well-being remained significant. The standardized regression coefficients beta in Model 5 showed that feelings and attitudes towards one's body and appearance had the strongest association with psychological wellbeing and the second strongest associations with psychological well-being were these with appearance satisfaction, peer victimization, gender and healthy eating. The two appearance self-attitudes variables explained the largest proportion of the variance in psychological well-being (several times larger) compared to the other groups of independent variables (Table 5). 
Table 5. Multiple Hierarchical Linear Regression of the Association between Appearance Self-attitudes and Psychological Well-being in Bulgarian Adolescents

\begin{tabular}{|c|c|c|c|c|c|c|c|c|c|c|c|c|c|c|c|}
\hline \multirow{2}{*}{ Independent variables } & \multicolumn{3}{|c|}{ Model 1} & \multicolumn{3}{|c|}{ Model 2} & \multicolumn{3}{|c|}{ Model 3} & \multicolumn{3}{|c|}{ Model 4} & \multicolumn{3}{|c|}{ Model 5} \\
\hline & $\mathrm{B}$ & SE & $\beta$ & B & SE & $\beta$ & $\mathrm{B}$ & SE & $\beta$ & $\mathrm{B}$ & SE & $\beta$ & $\mathrm{B}$ & SE & $\beta$ \\
\hline \multicolumn{16}{|l|}{ Appearance self-attitudes } \\
\hline Appearance satisfaction & $1.25^{\ddagger}$ & 0.16 & 0.18 & $1.11^{\ddagger}$ & 0.15 & 0.16 & $1.10^{\ddagger}$ & 0.15 & 0.16 & $1.09^{\ddagger}$ & 0.15 & 0.16 & $1.06^{\ddagger}$ & 0.15 & 0.15 \\
\hline $\begin{array}{l}\text { Feelings and attitudes } \\
\text { towards appearance }\end{array}$ & $0.40^{\ddagger}$ & 0.03 & 0.31 & $0.37^{\ddagger}$ & 0.03 & 0.28 & $0.37^{\ddagger}$ & 0.03 & 0.28 & $0.34^{\ddagger}$ & 0.03 & 0.26 & $0.31^{\ddagger}$ & 0.03 & 0.24 \\
\hline \multicolumn{16}{|c|}{ Socio-demographic characteristics } \\
\hline Gender & - & - & - & $-1.90^{\ddagger}$ & 0.26 & -0.14 & $-1.86^{\ddagger}$ & 0.26 & -0.14 & $-1.62^{\ddagger}$ & 0.27 & -0.12 & $-1.75^{\ddagger}$ & 0.26 & -0.13 \\
\hline Age & - & - & - & $-1.54^{\ddagger}$ & 0.25 & -0.11 & $-1.54^{\ddagger}$ & 0.25 & -0.11 & $-0.83^{\dagger}$ & 0.26 & -0.06 & $-1.00^{\ddagger}$ & 0.26 & -0.07 \\
\hline Family affluence & - & - & - & $0.25^{\ddagger}$ & 0.05 & 0.09 & $0.26^{\ddagger}$ & 0.05 & 0.10 & $0.20^{\ddagger}$ & 0.05 & 0.08 & $0.20^{\ddagger}$ & 0.05 & 0.07 \\
\hline Chronic conditions & - & - & - & - & - & - & $-1.16^{\dagger}$ & 0.41 & -0.05 & $-1.16^{\dagger}$ & 0.40 & -0.05 & $-1.01^{*}$ & 0.39 & -0.05 \\
\hline \multicolumn{16}{|c|}{ Health behaviours \& health outcomes } \\
\hline Healthy eating & - & - & - & - & - & - & - & - & - & $0.33^{\ddagger}$ & 0.05 & 0.13 & $0.33^{\ddagger}$ & 0.05 & 0.13 \\
\hline Physical activity & - & - & - & - & - & - & - & - & - & $0.32^{\ddagger}$ & 0.06 & 0.10 & $0.32^{\ddagger}$ & 0.06 & 0.10 \\
\hline Smoking & - & - & - & - & - & - & - & - & - & $-1.17^{\dagger}$ & 0.34 & -0.07 & $-1.09^{\dagger}$ & 0.33 & -0.06 \\
\hline Alcohol consumption & - & - & - & - & - & - & - & - & - & $-1.13^{\ddagger}$ & 0.26 & -0.08 & $-1.19^{\ddagger}$ & 0.26 & -0.09 \\
\hline Dieting to lose weight & - & - & - & - & - & - & - & - & - & 0.18 & 0.32 & 0.01 & 0.13 & 0.32 & 0.01 \\
\hline Overweight/obesity & - & - & - & - & - & - & - & - & - & $1.15^{\dagger}$ & 0.34 & 0.06 & $1.21^{\ddagger}$ & 0.34 & 0.07 \\
\hline Peer victimization & - & - & - & - & - & - & - & - & - & - & - & - & $-0.97^{\ddagger}$ & 0.12 & -0.14 \\
\hline \multicolumn{16}{|l|}{ Model summary } \\
\hline F total & \multicolumn{3}{|c|}{$257.45^{\ddagger}$} & \multicolumn{3}{|c|}{$131.28^{\ddagger}$} & \multicolumn{3}{|l|}{$111.08^{\ddagger}$} & \multicolumn{3}{|l|}{$72.61^{\ddagger}$} & \multicolumn{3}{|l|}{$73.70^{\ddagger}$} \\
\hline $\mathrm{R}$ & \multicolumn{3}{|l|}{0.43} & \multicolumn{3}{|l|}{0.47} & \multicolumn{3}{|l|}{0.48} & \multicolumn{3}{|l|}{0.53} & \multicolumn{3}{|l|}{0.54} \\
\hline Adjusted $\mathrm{R}^{2}$ & \multicolumn{3}{|l|}{0.18} & \multicolumn{3}{|l|}{0.22} & \multicolumn{3}{|l|}{0.22} & \multicolumn{3}{|l|}{0.27} & \multicolumn{3}{|l|}{0.29} \\
\hline$\Delta \mathrm{R}^{2}$ & \multicolumn{3}{|l|}{0.18} & \multicolumn{3}{|l|}{0.04} & \multicolumn{3}{|l|}{0.00} & \multicolumn{3}{|l|}{0.05} & \multicolumn{3}{|l|}{0.02} \\
\hline
\end{tabular}

${ }^{*} \mathrm{P}<0.05 ;{ }^{\dagger} \mathrm{P}<0.01 ;{ }^{\ddagger} \mathrm{P}<0.001 ; \mathrm{B}=$ The unstandardized regression coefficient; $\mathrm{SE}=$ The standard error of the regression coefficient; $\beta=$ The standardized regression coefficient; $\mathrm{F}=$ The test of overall model's significance; $\mathrm{R}=$ The multiple correlation coefficient; Adjusted $\mathrm{R}^{2}=$ The adjusted coefficient of multiple determination; $\Delta \mathrm{R}^{2}=$ The change in $\mathrm{R}^{2}$.

\section{Discussion}

The present study found that half of the Bulgarian adolescents were very satisfied with their overall appearance. Also, the mean values for the appearance self-attitudes variables were above the average for the scales, indicating mostly positive feelings and attitudes towards one's body and appearance and a general appearance satisfaction. Bulgarian adolescents' health and well-being seem to be thriving, as $95 \%$ of the participants rate their health as good or excellent (compared to HBSC average of $86 \%$ in the 13 -year old group and $83 \%$ in the 15 -year old group (27), and also, absence of or little psycho- somatic complaints and rather good psychological well-being were found.

It was confirmed that adolescents' more positive appearance self-attitudes were significantly associated with better self-rated health, absence of or little psychosomatic complaints and better psychological well-being. These associations remained significant even after they had been adjusted for socio-demographic characteristics of the participants, existence of chronic conditions, health behaviours and health outcomes, and peer victimization. Adolescents' appearance self-attitudes explained the largest proportions of the variances in self-rated health, psychosomatic complaints and psychological well-being, 
compared to the other groups of independent variables. Significant associations between appearance self-attitudes and measures of self-rated health, psychosomatic symptoms and mental health and wellbeing had been identified in several other studies conducted in recent years with adolescent samples globally (34-39).

The study found similar associations between the appearance self-attitudes and self-rated health, psychosomatic complain and psychological wellbeing, suggesting a universal relationship between adolescents' appearance self-attitudes and their health and well-being. However, regarding the covariate variables included in the regressions there were some noteworthy differences. For example, the three dependent variables had strongest associations with different covariates (for self-rated health, strongest association was with having chronic conditions; for psychosomatic complaints - with female gender, peer victimization, having chronic conditions, smoking and alcohol consumption; and for psychological well-being - with peer victimization, female gender and eating healthy). As might be expected, having chronic conditions and engaging in unhealthy behaviours like smoking and alcohol consumption were negatively associated with all health and well-being variables. Also, it was found that female gender and being older (15-year old group) were consistently associated with worse physical health and mental well-being. This is in line with the general tendencies regarding the effects of gender and age identified within the HBSC 2013/2014 study with respect to the variables selfrated health, multiple health complaints and lifesatisfaction (27). Similar patterns regarding gender and age were also found in other studies, exploring psychosomatic health and psychological well-being in adolescence (40-43). Peer victimization was important factor, contributing to more psychosomatic complaints and worse psychological well-being, but not to self-rated health. Healthy eating and physical activity were significantly associated with better self-rated health and better psychological wellbeing, but not with psychosomatic complaints. Dieting to lose weight was associated only with having more psychosomatic complaints. Surprisingly, being overweight/obese was associated with better psychological well-being and absence of or little psychosomatic complaints. Higher family affluence was associated with better self-rated health and better psychological well-being, but with more psychosomatic complaints. Analyses of international samples within the HBSC 2013/2014 study $(27,44)$ and within four cycles of the HBSC study (45) indicated that higher family affluence was consistently associated with better self-rated health and higher life satisfaction, but findings for psychosomatic complaints were mixed. Higher family affluence was associated with more psychosomatic complaints in about a third of the countries for boys and in about a half of the countries for girls in the HBSC 2013/2014 study (27).

Among the strengths of the study is that it is based on a national representative sample of Bulgarian adolescents, which allows generalizability of findings to the Bulgarian adolescent population. A strength of the study is that it uses methodology for the assessment of appearance self-attitudes and health and well-being that has been internationally validated through the long-standing experience of the HBSC research network. Also, a large number of covariates were controlled for in the multiple hierarchical linear regressions. The study added valuable knowledge to the rarely explored topic of the associations between appearance self-attitudes and general health and well-being in adolescence.

\section{Limitations of the Study}

A limitation of this study is its cross-sectional design, which does not allow conclusions regarding the direction of causality between appearance selfattitudes and health and well-being to be made. It is possible that existing physical or mental health problems and/or side effects of medical treatment negatively impact physical appearance and its subjective experience. Another limitation of the study is that indicators for health are self-reported and not measured by objective indicators. Among the limitations of the study is that it does not inves- 
tigate the role of potential confounders in the appearance - health and well-being association like personality factors, the importance of appearance for the self-concept and global self-esteem, etc.

\section{Conclusion}

The study found that appearance satisfaction and positive feelings and attitudes towards one's body and appearance were significantly associated with better self-rated health, absence of or little psychosomatic complaints and better psychological wellbeing in a representative sample of Bulgarian adolescents. Moreover, these associations remained significant after the addition of a large number of covariates, including adolescents' socio-demographic characteristics such as gender, age and socioeconomic status; existing chronic health conditions; health behaviours such as healthy eating, physical activity, smoking, alcohol consumption, and dieting to lose weight; overweight/obesity; and peer victimization. Adolescents' appearance self-attitudes explained the largest proportions of the variances in self-rated health, psychosomatic complaints and psychological well-being, compared to the other groups of independent variables. These results demonstrate the great significance of appearance self-attitudes for physical and mental health and well-being in adolescence. They are important for medical practice, where clinicians should consider negative appearance self-attitudes as risk factors for adolescent health. The study has implications also for health education and health promotion among adolescent populations, where the topic of appearance selfperceptions and self-attitudes and the development of positive body image should be given considerable attention. An important goal would also be to raise awareness within society about the significance of appearance self-attitudes for physical and mental health and well-being in adolescence as well as to promote healthier body and appearance ideals.

Acknowledgements: The 2013/2014 HBSC study in Bulgaria was supported by the Ministry of Education and Science of Bulgaria through funding of data collection.
Conflict of Interest: The author declares that she has no conflict of interest.

\section{References}

1. Cash TF. Preface. In: Cash TF, editor. Encyclopedia of body image and human appearance. Elsevier - Academic Press; 2012. p. XIX-XX.

2. Alexandrova-Karamanova A. Appearance in young people: between health and success [in Bulgarian]. Vanshniyat vid pri mladite hora: mezhdu zdraveto i uspeha. Sofia: DioMira; 2019.

3. Grogan S. Body image: Understanding body dissatisfaction in men, women, and children, 3rd edition. London; New York: Routledge, 2017.

4. Smolak L. Body image development in childhood. In: Cash TF, Smolak L, editors. Body image: A handbook of science, practice, and prevention, 2nd edition. New York: Guilford Press, 2011. p. 67-75.

5. Tiggemann M. Sociocultural perspectives on human appearance and body image. In: Cash TF, Smolak L, editors. Body image: A handbook of science, practice, and prevention, 2nd edition. New York: Guilford Press, 2011. p. 12-19.

6. Rodin J, Silberstein L, Striegel-Moore R. Women and weight: a normative discontent. In: Sonderegger TB, editor. Nebraska Symposium on motivation, vol. 32, Psychology and Gender. Lincoln: University of Nebraska Press; 1985. p. 267-307.

7. Levine M, Smolak L. Body image development in adolescence. In: Cash TF, Pruzinsky T, editors. Body Image: A Handbook of theory, research and clinical practice New York: The Guilford Press; 2002. p.74-82.

8. Bucchianeri MM, Arikian AJ, Hannan PJ, Eisenberg ME, Neumark-Sztainer D. Body dissatisfaction from adolescence to young adulthood: findings from a 10-year longitudinal study. Body image. 2013;10(1):1-7.

9. Kim D-S, Kim H-S. Body-image dissatisfaction as a predictor of suicidal ideation among Korean boys and girls in different stages of adolescence: a two-year longitudinal study. Journal Adolesc Health. 2009;45:47-54.

10. Wang SB, Haynos AF, Wall MM, Chen C, Eisenberg ME, Neumark-Sztainer D. Fifteen-year prevalence, trajectories, and predictors of body dissatisfaction from adolescence to middle adulthood. Clin Psychol Sci. 2019;7(6):1403-15.

11. Brown CL, Skelton JA, Perrin EM, Skinner AC. Behaviors and motivations for weight loss in children and adolescents. Obesity. 2016;24:446-52.

12. Ojala K, Vereecken C, Valimaa R, Currie C, Villberg J, Tynjala J, Kannas L. Attempts to lose weight among over- 
weight and non-overweight adolescents: a cross-national survey. Int J Behav Nutr Phys Act. 2007;4:50.

13. National Institute on Drug Abuse - NIDA. Steroids and other appearance and performance enhancing drugs (APEDs) [research report on the Internet]. Bethesda: NIDA; 2018 Feb 21 [cited 2018 Mar 26]. Available from: https://www.drugabuse.gov/publications/research-reports/ steroids-other-appearance-performance-enhancing-drugsapeds/what-are-different-types-apeds

14. Canadian Pediatric Society. Dieting in adolescence. Paediatr Child Health. 2004;9:487-91.

15. Crow S, Eisenberg ME, Story M, Neumark-Sztainer D. Suicidal behaviour in adolescents: relationship to weight status, weight control behaviours and body dissatisfaction. Int J Eat Disord. 2008;41:82-7.

16. Neumark-Sztainer D, Hannan PJ. Weight-Related behaviors among adolescent girls and boys results from a national survey. Arch Pediatr Adolesc Med. 2000;154:569-77.

17. Ditch the Label. The annual bullying survey 2015 [research report on the Internet]. Brighton: Ditch the Label; 2015 [cited 2017 Sep 20]. Available from: https://www. ditchthelabel.org/research-papers/the-annual-bullyingsurvey-2015/

18. Bucchianeri MM, Gower AL, McMorris BJ, Eisenberg ME. Youth experiences with multiple types of prejudicebased harassment. J Adolesc. 2016;51:68-75.

19. Oliveira WA, Silva MAI, Mello FCM, Porto DL, Yoshinaga ACM, Malta DC. The causes of bullying: results from the National Survey of School Health (PeNSE). Rev Lat Am Enfermagem. 2015;23:275-82.

20. Goldfield G, Moore C, Henderson K, Buchholz A, Obeid $\mathrm{N}$, Flament $\mathrm{M}$. The relation between weight-based teasing and psychological adjustment in adolescents. Paediatr Child Health. 2010;15(5):283-88.

21. Neumark-Sztainer D, Falkner N, Story M, Perry C, Hannan PJ, Mulert S. Weight-teasing among adolescents: correlations with weight status and disordered eating behaviors. Int J Obes Relat Metab Disord. 2002;26:123-31.

22. Higgins S, Wysong A. Cosmetic surgery and body dysmorphic disorder - an update. Int J Womens Dermatol. 2017;4(1):43-8.

23. Harvey K. Cosmetic procedures: demand, motivations and influencing factors [research report on the Internet]. London: Nuffield Council on Bioethics; 2016 [cited 2019 Oct 2]. Available from: http://nuffieldbioethics.org/ wp-content/uploads/Cosmetic-procedures-Motivationsreview.pdf

24. National Academies of Sciences, Engineering, and Medicine. Preventing bullying through science, policy, and practice. Washington, DC: The National Academies Press; 2016.

25. Griffiths S, Murray SB, Bentley C, Gratwick-Sarll K, Harrison C, Mond JM. Sex differences in quality of life impairment associated with body dissatisfaction in adolescents. J Adolesc Health. 2017;61(1):77-82.

26. Ren L, Xu Y, Guo X, Zhang J, Wang H, Lou X, Liang J, Tao F. Body image as risk factor for emotional and behavioral problems among Chinese adolescents. BMC Public Health. 2018 Oct 16;18(1):1179.

27. Inchley J, Currie D, Young T, Samdal O, Torsheim T, Augustson L, et al., editors. Growing up unequal: gender and socioeconomic differences in young people's health and well-being. Health Behaviour in School-aged Children (HBSC) study: International report from the 2013/2014 survey. Health Policy for Children and Adolescents, No 7 , World Health Organization; 2016.

28. Currie C, Inchley J, Molcho M, Lenzi M, Veselska Z, Wild F, editors. Health Behaviour in School-Aged Children (HBSC) study protocol: background, methodology and mandatory items for the 2013/14 survey. St. Andrews: Child and Adolescent Health Research Unit, University of St. Andrews; 2014.

29. Orbach I, Mikulincer M. Body investment scale: Construction and validation of a body experience scale. Psychol Assess. 1998;10(4):415-25.

30. Ravens-Sieberer U \& the European KIDSCREEN group. The KIDSCREEN questionnaires. Quality of life questionnaires for children and adolescents - handbook. Lengerich: Pabst Science Publisher; 2006.

31. Kidscreen.org: Project. [webpage on the Internet]. Hamburg: Kidscreen.org; 2011 [cited 2019 Dec 8]. Available from: https://www.kidscreen.org/english/project/

32. Torsheim T, Cavallo F, Levin KA, Schnohr C, Mazur J, Niclasen B, Currie C \& the FAS Development Study Group. Psychometric validation of the revised Family Affluence Scale: a latent variable approach. Child Indic Res. 2016;9:771-84.

33. Cole TJ, Bellizzi MC, Flegal KM, Dietz WH. Establishing a standard definition for child overweight and obesity worldwide: international survey. Br Med J. 2000;320:1-6.

34. Silva PD, Guimarães JM, Griep RH, Melo EC, Matos SM, Molina MD, Barreto SM, Fonseca MD. Association between body image dissatisfaction and self-rated health, as mediated by physical activity and eating habits: Structural equation modelling in ELSA-Brasil. Int J Environ Res Public Health. 2018;15:790.

35. Kim M, Lim Y, Kwak H. Dietary behaviors and body image recognition of college students according to the selfrated health condition. Nutr Res Pract. 2008;2:107-13. 
36. Altinok A, Kara A. Relationship between body image, psychological symptom level and interpersonal style: alternative models. Dusunen Adam. 2017; 30:170-80.

37. Sujoldžić A, De Lucia A. A cross-cultural study of adolescents - BMI, body image and psychological well-being. Coll Antropol. 2007;31(1):123-30.

38. Lemes DC, Câmara SG, Alves GG, Aerts DR. Body image satisfaction and subjective wellbeing among ninth-grade students attending state schools in Canoas, Brazil. Cien Saude Colet. 2018;23(12):4289-98.

39. Delfabbro P, Winefield AH, Anderson S, Hammarström A, Winefield HR. Body image and psychological wellbeing in adolescents: the relationship between gender and school type. J Genet Psychol. 2011;172(1):67-83.

40. Meade T, Dowswell E. Adolescents' health-related quality of life (HRQoL) changes over time: a three year longitudinal study. Health Qual Life Outcomes. 2016;14:1-8.

41. Booker CL., Kelly YJ, Sacker A. Gender differences in the associations between age trends of social media interaction and well-being among 10-15 year olds in the UK. BMC Public Health. 2018;18:1-12.

42. Patalay P, Fitzsimons E. Development and predictors of mental ill-health and wellbeing from childhood to adolescence. Soc Psychiatry Psychiatr Epidemiol. 2018;53:131123.

43. Simonsson B, Nilsson KW, Leppert J, Diwan VK. Psychosomatic complaints and sense of coherence among adolescents in a county in Sweden: a cross-sectional school survey. Biopsychosoc Med. 2008;2:4.

44. Torsheim T, Nygren JM, Rasmussen M, Arnarsson AM, Bendtsen P, Schnohr CW, Nielsen L, Nyholm M. Social inequalities in self-rated health: a comparative cross-national study among 32,560 Nordic adolescents. Scand J Public Health. 2018;46(1):150-56.

45. Chzhen Y, Moor I, Pickett W, Toczydlowska E, Stevens G. Family affluence and inequality in adolescent health and life satisfaction: evidence from the HBSC study 2002 2014. Innocenti Working Paper No.2016-10. Florence: UNICEF Office of Research; 2016. 